\title{
Automated Image Analysis Using a Tabletop Low Voltage TEM
}

\author{
Gustaf Kylberg $^{1}$, Ida-Maria Sintorn ${ }^{1,2}$, Martin Ryner ${ }^{1}$, Mathieu Colomb-Delsuc ${ }^{1}$ \\ 1. Vironova AB, Stockholm, Sweden \\ 2. Uppsala University, Uppsala, Sweden
}

The development of technologies using nanoparticles has been significantly increasing over the past decade, with applications in a broad scope of fields, as well in material sciences as in life sciences, and the demand on particle characterization has been growing accordingly. Amongst the methods available for particle characterization, Electron Microscopic technologies in contrast to indirect methods like Dynamic Light Scattering and Nano Tracking Analysis result in high resolution images that provide morphological information and particle identification. EM however requires an experienced operator and can be time and resource consuming. A method allowing to simplify the analysis process with an automated image acquisition of samples as well as an automatic image analysis on a low-voltage electron microscope is proposed herein.

Such automation capabilities prove relevant for example in the domain of the vaccine industry, where new requirements from the United States Pharmacopeial Convention (USP) and the U.S. Food and Drug Administration (FDA) put pressure for root cause analysis to understand impact on changes during process and product development. The new demands involves morphological characterization of the particles present in the formulations, typically nanoparticles like viruses, virus-like particles and adjuvants in the range of $0.01-0.1 \mu \mathrm{m}$. When entering production phases of such products, the high throughput of specimen to be analyzed can be a limiting factor for electron microscopy to be used as a method of choice. We therefore introduced a process that can allow a rapid and efficient characterization of specimen, using a low voltage instrument, the MiniTEM ${ }^{\mathrm{TM}}$.

The instrument is a system based on the LVEM 25 desktop sized microscope from Delong Instruments A.s. The low voltage $(25 \mathrm{keV})$ usage allows the system to be placed in any lab or office area, and the simplify design of the microscope makes its use easier to apprehend for users with limited experience in electron microscopy.

A case study involving the characterization of biological nanoparticles in which the specimen was automatically analyzed will be discussed (Figure 1). Detected particles within a preset size range are, while screening, automatically measured and characterized based on assigned attributes for each type of particle. If desired the screening can be set to stop when sufficient information has been acquired. Stop criteria such as a minimum number of detected particles or statistical stability of the size distribution can be used. 

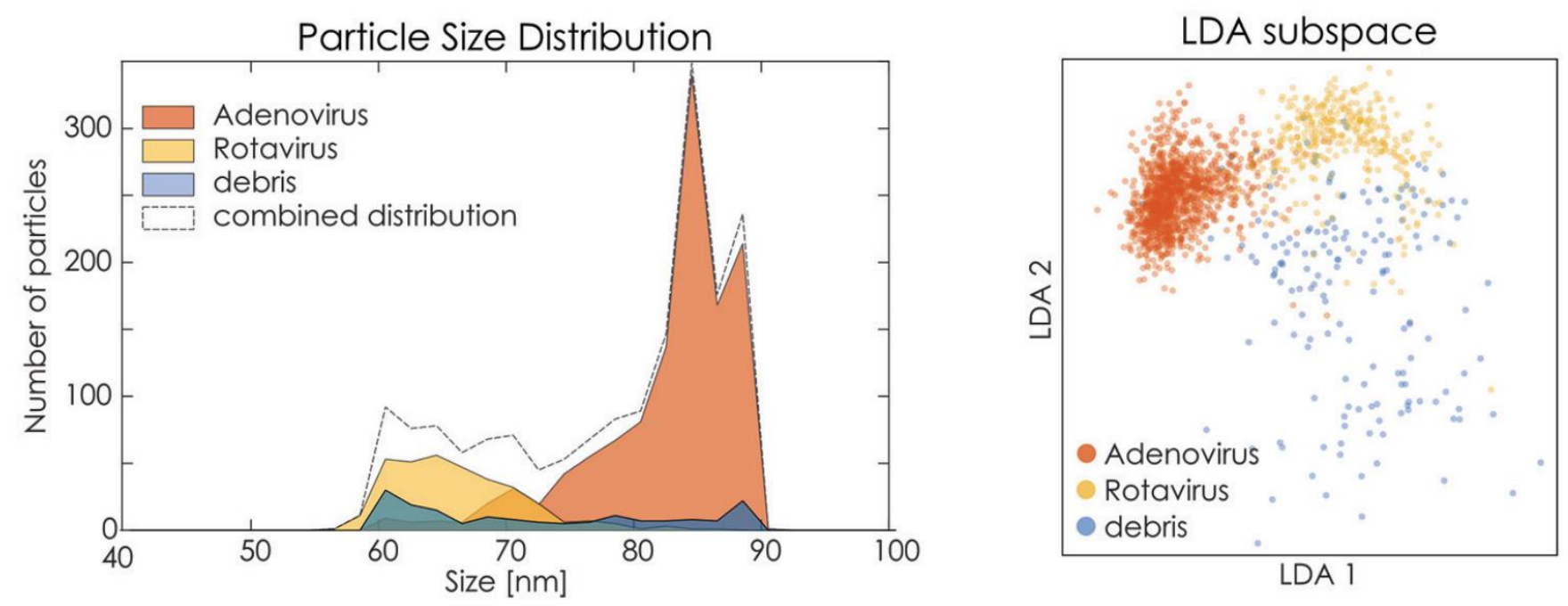

Figure 1. Typical study of a specimen containing a mixture of adenoviruses and rotaviruses, as well as some amount of debris. Left: Particle size distribution of the specimen with discriminate classification depending on the particle type. Right: Visualization of the feature space using linear discriminant analysis (LDA) showing distinct cluster for each particle type. 